\title{
FEDC
}

\section{Using Unmanned Aircraft System (UAS) and Satellite Imagery to Map Aquatic and Terrestrial Vegetation}

Sean Griffin and Kristofer Lasko

PURPOSE: The purpose of this study is to demonstrate the application potential of using unmanned aerial systems (UAS) combined with a time series of moderately high-resolution satellite imagery for mapping ecological restoration progress and resulting land cover changes. This technical note addresses a project under the US Army Corps of Engineers Ecosystem Management and Restoration Research Project (EMRRP) focusing on image acquisition and assessment, digital image processing techniques, analytical methodology, geospatial product development, and documentation of best practice for future data acquisition and analysis in support of ecological management efforts.

BACKGROUND: This investigation has been developed by the US Army Engineer Research and Development Center (ERDC) Geospatial Research Lab (GRL) in response to a Statement of Need (SON) presented to the 2017 Environmental Research Area Review Group entitled "Evaluation and Optimization of Unmanned Aircraft Systems to Sense, Identify, and Map in Aquatic Systems" (2017-ER-17). Monitoring the distribution of vegetative cover is an integral element in the planning and implementation of both small and large ecosystem restoration projects. Facilitating the expansion of desirable native species, while suppressing the spread of undesirable exotic plants, is critical to restore both aquatic and terrestrial landscapes. Federal, state and local land management agencies need new techniques to monitor and manage ecosystem vegetation communities at multiple spatio-temporal scales (Reif and Theel 2017). Recent advancements in UAS provide high spatial, spectral, and temporal resolution aerial imagery to accurately and precisely monitor the distribution of plant communities. However, UAS have limitations, including (1) restricted areal coverage $(<5,000$ acres) by most light UAS vehicles, (2) a lack of spectral bands (e.g., near-infrared and shortwave infrared) needed to accurately differentiate plant species, and (3) substantial data processing and workflow challenges associated with very high spatial resolution $\left(<5 \mathrm{~cm}^{* \dagger}\right.$ pixels $)$ image mosaics. This proposed investigation will address these limitations by integrating satellite multispectral imagery into the vegetation monitoring workflow, demonstrating the critical requirement to deploy UAS sensors that acquire imagery beyond the visible wavelengths and employing best practices in image processing and orthorectification, as well as building practical software tools that simplify the post-processing and classification of multi-band image mosaics. The

\footnotetext{
* For a full list of the spelled-out forms of the units of measure used in this document, please refer to US Government Publishing Office Style Manual, 31st ed. (Washington, DC: US Government Publishing Office 2016), 248-52, https://www.govinfo.gov/content/pkg/GPO-STYLEMANUAL-2016/pdf/GPO-STYLEMANUAL-2016.pdf.

${ }^{\dagger}$ For a full list of the unit conversions used in this document, please refer to US Government Publishing Office Style Manual, 31st ed. (Washington, DC: US Government Publishing Office 2016), 345-7, https://www.govinfo.gov/content/pkg/GPO-STYLEMANUAL-2016/pdf/GPO-STYLEMANUAL-2016.pdf.
} 
knowledge gained in this investigation will not only be transitioned as practical tools and workflows that build vegetation distribution maps in support of ecosystem management and restoration but also used to guide future best practices in image processing for land cover mapping.

The objectives of this study are to develop and test multistage ecosystem monitoring methods that incorporate the following:

1. Remotely sensed optical imagery, from both spaceborne and airborne sensors of varying spatial resolutions

2. Limited ground reference data combined with a statistically-robust accuracy assessment

3. Commercial software image processing toolkits (ESRI ArcMap and Harris ENVI).

This is a first-phase study that primarily leveraged tools developed in the ENVI image analysis software as an Army solution; however, future tool development will be primarily applied to the ArcMap environment given the availability of Environmental Systems Research Institute (ESRI)licensed products within USACE District Geographic Information System (GIS) shops.

STUDY AREA: The study area is located in south-central Florida and consists of three sites designated for long-term landscape remediation and monitoring by the US Department of Agriculture, National Resource Conservation Service, and the US Army Corps of Engineers (USACE) Jacksonville District Ecosystem Restoration Program (Figure 1). The sites are on a predominantly treeless landscape referred to as dry prairie that is composed of grassland, shrubland, sandy ridges, and mixed forest islands. Historically, dry prairie was maintained by frequent fires and flooding from the nearby Kissimmee River system. Most of the landscape today has been converted to improved pasture for cattle and pine plantation, and primary ecological functions have been altered for flood control, leading to the decline and endangered species status of over 50 species of flora and fauna. In response, public agencies and private groups have sought to protect the landscape through long-term land acquisition, conservation, and restoration programs (Griffin et al. 2011). The common objective is to return converted land to dry prairie, manage for invasive species, and monitor short- and long-term landscape change in relation to ecological restoration. 


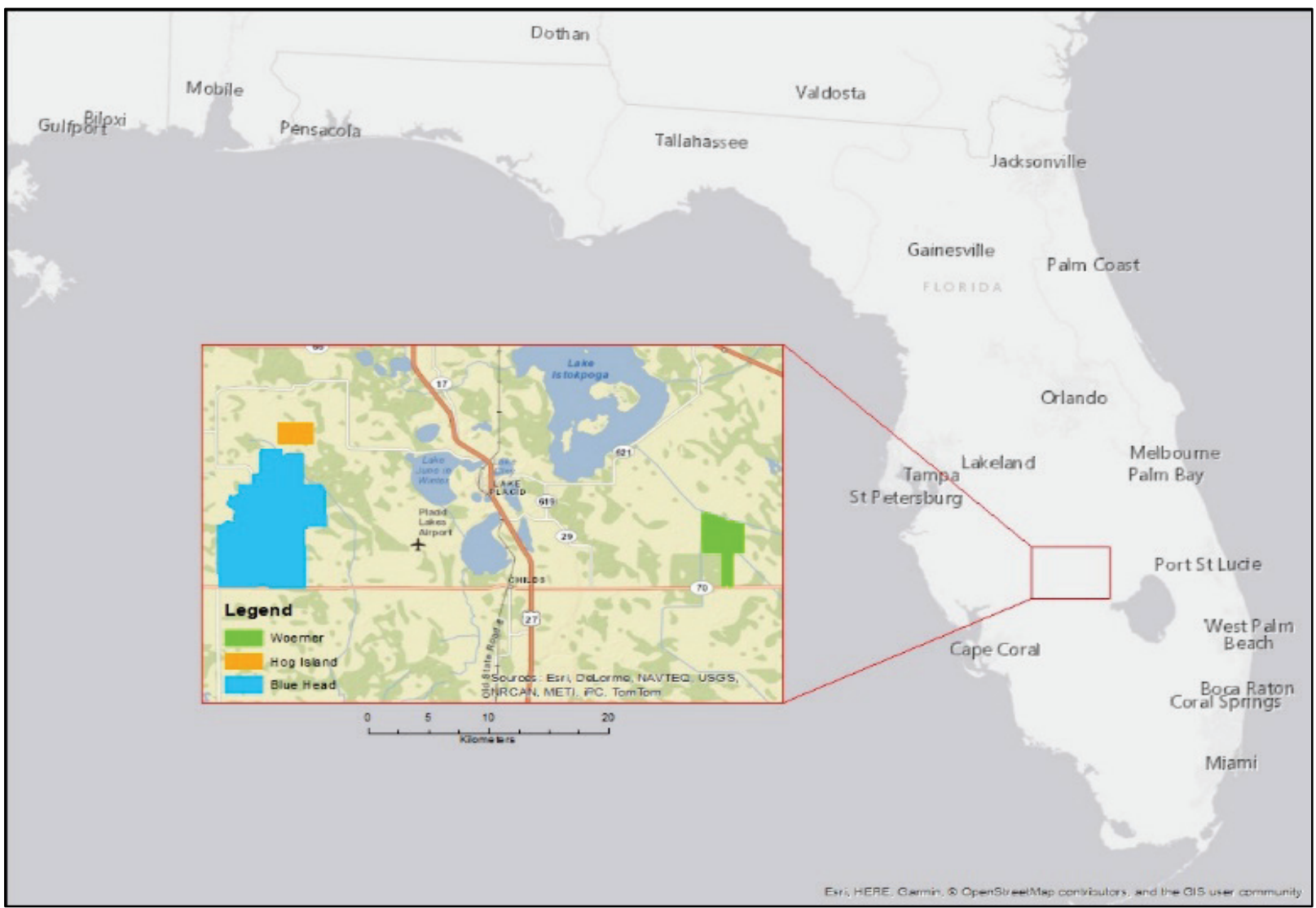

Figure 1. Overview map of study area.

The three study sites include the following:

\section{Woerner}

Woerner occupies a land area of approximately $13 \mathrm{~km}^{2}$ with a landscape characterized by dry prairie, perennial wetlands, small irrigation canals, sod farms, orchards, grasslands, and open forest (Figure 2). A large channelized river borders at the north end of the study area. The study area lies at approximately latitude N81.18o and longitude W27.25o.

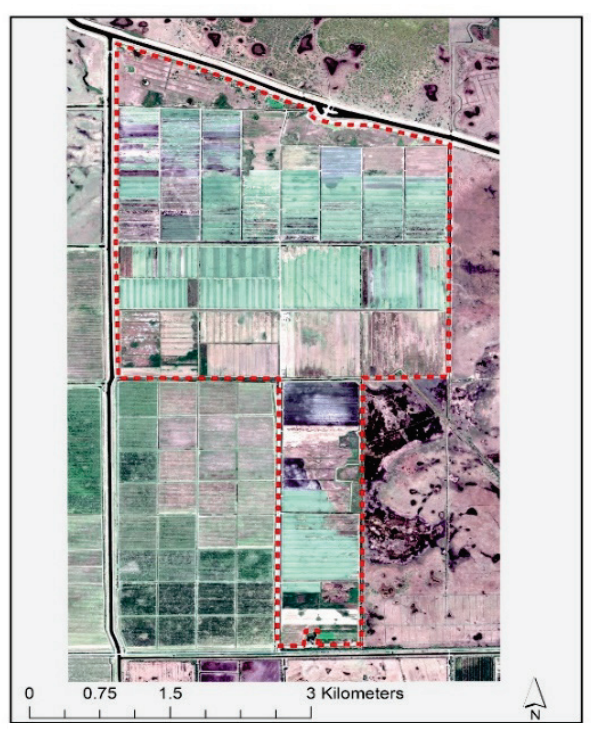

Figure 2. Pleiades coverage of Woerner, 11 February 2015. 


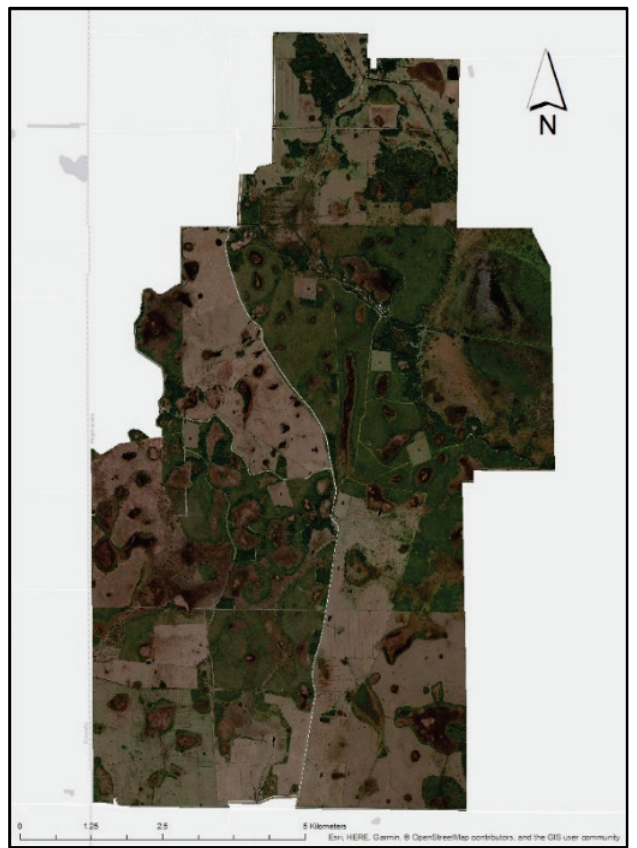

Figure 3. WorldView-2 coverage of Blue Head, 14 February 2015.

(C) 2015 DigitalGlobe NextView License

\section{Hog Island}

Hog Island covers an approximate area of $5 \mathrm{~km} 2$ between latitudes $\mathrm{N} 27.34 \mathrm{o}$ and longitude W81.51o. The site is predominantly covered with improved pasture, mixed forest hammocks, perennial wetland, and open water (Figure 4).

\section{Blue Head}

Blue Head covers an approximate area of $75 \mathrm{~km}^{2}$ between latitudes N27.260 and longitude W81.520. The site is predominantly covered with dry prairie, improved pasture, mixed forest hammocks, and perennial wetland (Figure 3).

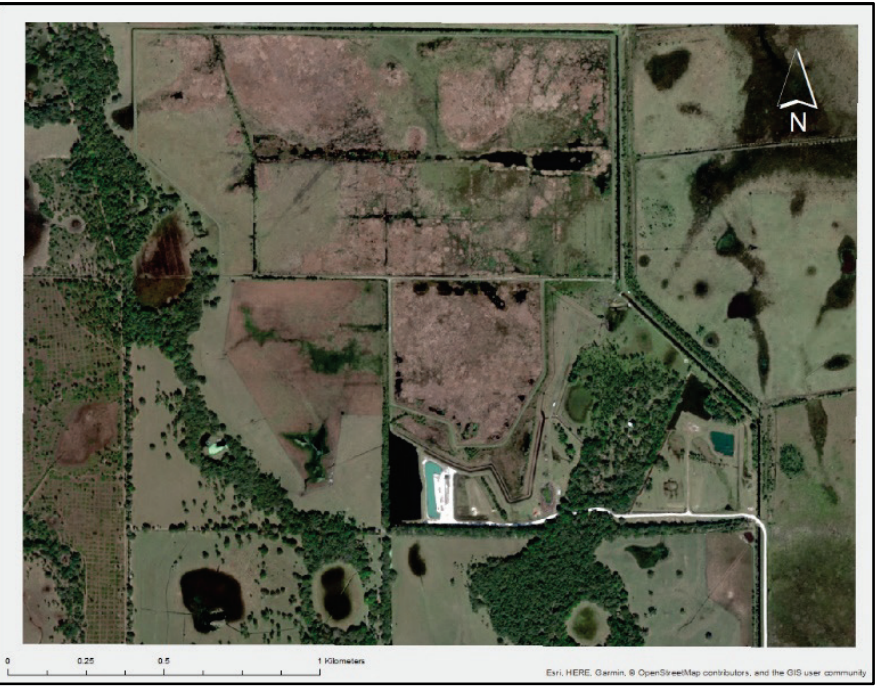

Figure 4. WorldView-2 coverage of Hog Island, 14 February 2015.

(C) 2015 DigitalGlobe NextView License 


\section{DATA SOURCES}

Satellite imagery. Archived high spatial resolution, multispectral imagery data are available through the Maxar Global Enhanced GEOINT Delivery (G-EGD). G-EGD is freely available to individuals with a current Department of Defense Public Key Infrastructure Common Access Card and provides direct user access to digital holdings of all DigitalGlobe sensors and a variety of foreign sensor assets. However, access to the continental United States or domestic coverage requires approval by the Army Department Requirements Office. An alternative to G-EGD is providing a written request to the Army Geospatial Center Imagery Office (AIO). In addition to archived imagery, the AIO can submit new imagery collection tasks on request.

This study acquired imagery through G-EGD with Airbus Defence \& Space Pleiades-1A and DigitalGlobe WorldView-2 imagery data fitting the temporal, spatial, and spectral criteria required to perform land cover mapping and multi-temporal analysis with emphasis on monitoring change in vegetation and water cover. In addition, all images were acquired during the fall-winter months to prevent seasonal bias when determining true change but primarily to avoid cloud contamination during the wetter months when clear electro-optical images are not possible.

Pleiades and WorldView-2 have comparable spatial resolution of $50 \mathrm{~cm}$ panchromatic and $2.0 \mathrm{~m}$ multispectral; however, WorldView-2 has a higher spectral resolution with the addition of four bands that include a coastal $(410-450 \mathrm{~nm})$, yellow $(585-625 \mathrm{~nm})$, red edge $(705-745 \mathrm{~nm})$, and second near-infrared $(860-900 \mathrm{~nm})$, the latter two are important for discriminating amongst vegetation types.

Image acquisition dates for each study site are shown in the appendix.

\begin{abstract}
Unmanned Aerial Systems (UAS) imagery. Very high spatial resolution (VHR) imagery data were collected over all three study sites during the months of January and May 2015 using a custom fixedwing platform developed by the University of Florida (now designated the Altavian Nova F6500) with the payload consisting of a Canon EOS Rebel T2i Digital Single-lens reflex camera. The UAS provided true color images that were orthorectified and mosaicked using Agisoft Photoscan 3D mapping and modeling software (Figure 5). Color infrared imagery critical for image classification was only collected for the Hog Island site.
\end{abstract}

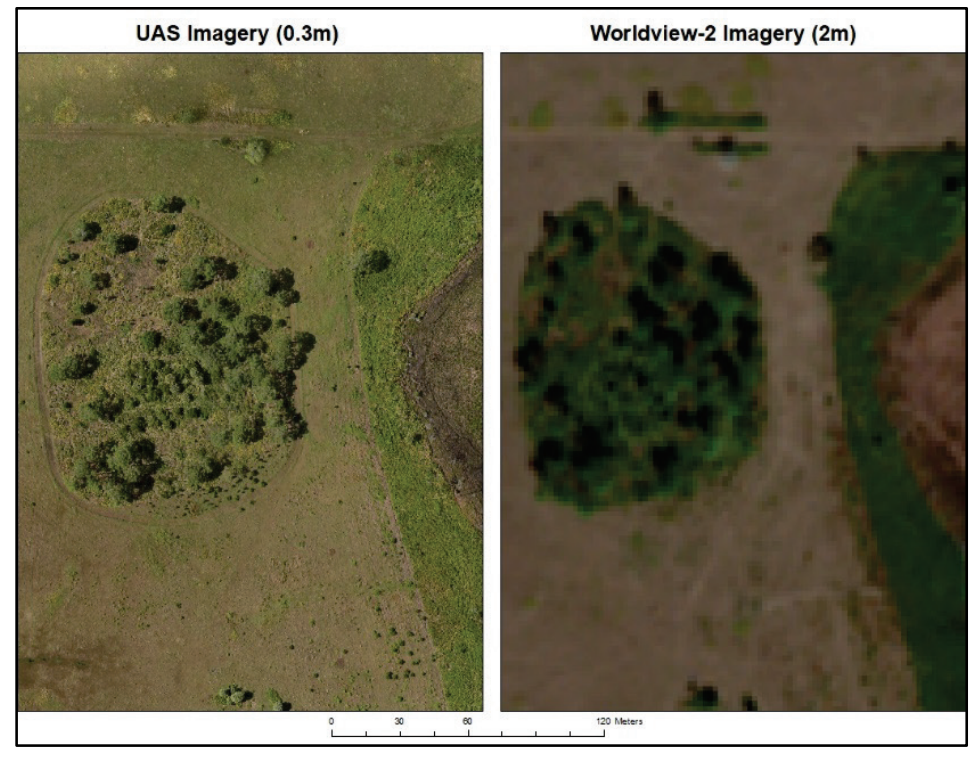

Figure 5. Comparison of spatial resolution between VHR airborne and HR Worldview-2 satellite imagery over Hog Island.

(c) 2015 DigitalGlobe NextView License 
METHODS: The majority of effort for most remote sensing analysis projects involves data preparation. These steps are critical to standardizing data used in multi-temporal change detection (Manjula et al. 2013). Digital image preprocessing can be a manually intensive commitment, but the routines can be easily coded into programmatically enabled toolkits.

The ERDC GRL has developed the Enhanced Terrain Processing (ETP) Tools, an image analysis toolkit that facilitates preprocessing through a suite of automated routines developed specifically as add-ons for the ESRI ArcMap geospatial processing software and Harris ENVI image analysis software. Currently, the preprocessing workflow of ETP tools is only applicable to satellite sensors, but future development will include UAS routines. The workflow for UAS and satellite imagery is shown in Figure 6. More detail about preprocessing for satellite imagery, such as radiometric and geometric correction, is shown in the appendix.

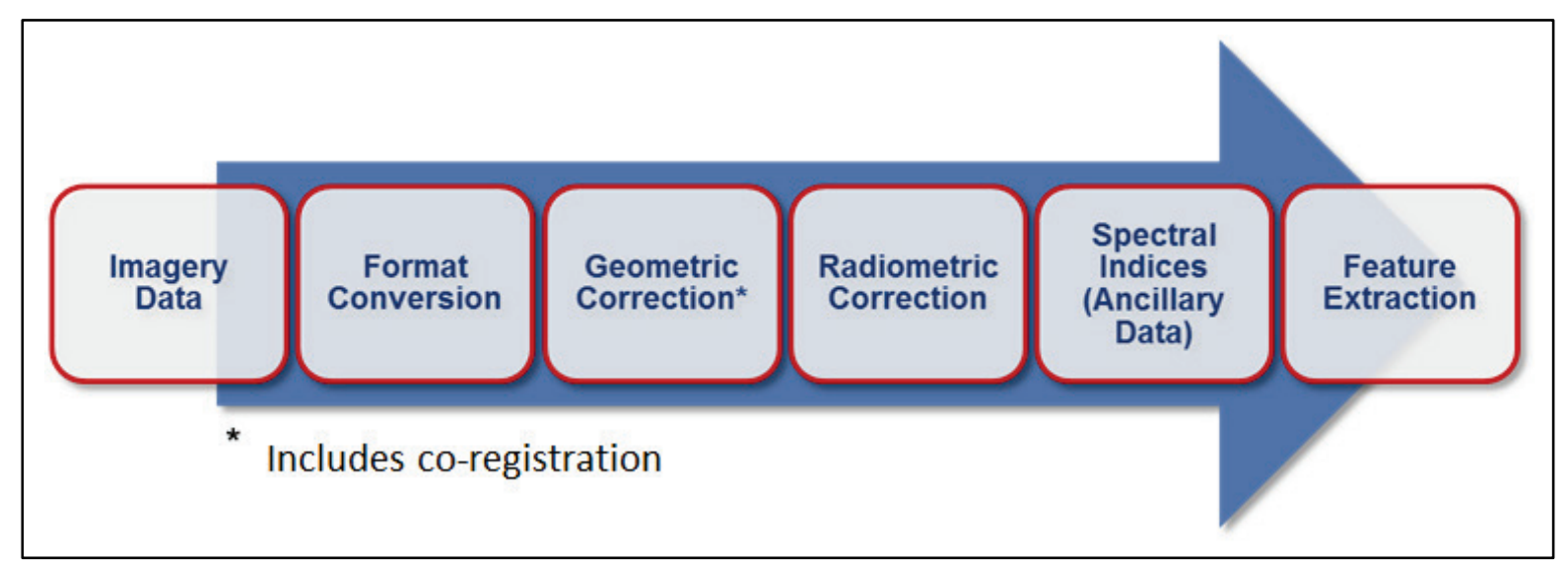

Figure 6. ETP Tools image processing workflow.

Data Processing and Feature Extraction. Feature extraction is a technique to classify information into mutual relationships within an image region. In the context of ETP Tools and EMRRP, feature extraction includes both low-level information extraction using pixel-based classification methods and high-level information that includes the complexity of image regions such as color, size, topology, and texture of an object. This study focused primarily on pixel-wise classifiers to derive final map products, but the ETP Tools provide the functions for robust object-based imagery analysis.

Land cover classes were mapped following a supervised classification approach where training data were collected over each site based on the UAS imagery supplemented by the respective WorldView2 and Pleiades time-series of imagery. In this case, a pixel-based classifier was employed for land cover mapping at the test sites using the Support Vector Machine machine-learning algorithm. Nonparametric machine learning classifiers have been proven to outperform traditional, parametric classifiers such as maximum likelihood because machine learners have no prior data distribution assumptions, generally unaffected by noisy data, and provide greater separability of spectrally similar land cover types (Mondal et al. 2012). Due to the unique land cover and restoration at each site, different mapping foci were demonstrated. For Blue Head and Hog Island, Anderson Level II land cover classes were used (https://landcover.usgs.gov/classes.php). These include classes such as permanent and seasonal water, wetlands, barren, etc. Change between imagery of different dates is accomplished based on a change detection assessment and thematic change workflow in the toolbox. 
For the Woerner site, change was compared between vegetated land and water, water to vegetated land, and areas that remained constant between winter 2010 and 2017. In other applications, analysts can select land cover types of importance to a particular mission.

The robust accuracy assessment demonstrated in this study is an important step in land cover mapping applications. After developing the 2010-2017 land cover change map, a stratified random sample was generated based upon the proportion of each land cover change class and following multinomial probability sampling (Congalton and Green 2008). To ensure quality control, the accuracy assessment must be represented by a statistically significant sample size and a confusion matrix that provides meaningful values for comparison such as producer's, user's, and overall map accuracies. The analysis is then taken a step further than most land cover applications by adjusting the confusion matrix and aforementioned accuracy metrics based upon unbiased area proportions of the randomly sampled validation data points. The unbiased area proportions essentially account for the number of validation points relative to the land cover class proportions in the map. Consequently, the new metrics improve measures of accuracy, provide confidence intervals for each metric, and derive unbiased areal estimates for each land cover class. These land cover change area estimates adjust the land area for each class (e.g., water to vegetation) based on the results of the accuracy assessment (Olofsson et al. 2014; Kontgis et al. 2015; Lasko et al. 2018). Much of the workflow for accuracy assessment can be incorporated into the toolbox.

UAS imagery. As previously noted, traditional data collection methods for long-term, broad-scale ecological research can be expensive, time consuming, and not possible in difficult terrain or inaccessible areas. UAS data are also collected at a spatial scale that complements field data collection. Unlike high spatial resolution satellite imagery, the resolution of $3.5 \mathrm{~cm}$ or better from UAS has high enough fidelity to distinguish plant communities and sometimes species, which makes aerial surveillance and monitoring of invasive species a possibility. Furthermore, UAS collect overlapping imagery with high precision spatial accuracy allowing photogrammetric solutions to be applied in three-dimensional (3D) mapping software. This provides an affordable terrain elevation solution compared to Light Detection and Ranging (LiDAR) and can significantly improve map classification accuracies with the addition of height information. Classified maps derived from UAS can also serve as a surrogate set of field-training data for satellite-derived map classifications and image-based ground reference for assessing map accuracy.

For this study, the UAS imagery was post-processed using Agisoft Photoscan, a commercial 3D modeling and mapping software with an automated photogrammetry pipeline used to orthorectify, mosaic, and estimate 3D information from 2D images by generating dense point clouds and digital surface models (DSM) and digital terrain models. Unfortunately, the UAS data collected over the three Florida sites did not meet the spectral or post-processing criteria needed to develop high-accuracy maps. The lack of a near-infrared (NIR) band for the Woerner and Blue Head sites ruled out vegetation analysis and the DSM developed for all sites was post-processed with low to medium quality resulting in an elevation model with multiple data anomalies and artifacts. It is recommended in future studies to emphasize the need for a true multiband spectral sensor (e.g., Micasense Sequoia) and either LiDAR-derived elevation data or high-quality post-processed electro-optical point clouds.

RESULTS: A land cover map and normalized difference vegetation index (NDVI) change detection analysis were performed for each study site. The NDVI is a simple image transformation that takes advantage of the red light absorption and NIR reflection of leaf pigments and normalizes 
the values between 1.0 and -1.0 (Tucker 1979). Healthy, green vegetation will have high positive values, and stressed or senesced vegetation will have low values. An accuracy assessment was conducted to quantify not only the accuracy of mapped classes but also to understand which classes were underestimated or overestimated. It is also shown how the statistically robust accuracy assessment might compare with other procedures. Last, based on the accuracy assessment, there is an adjustment to each of the metrics and unbiased areal estimates generated for each change class. Unbiased areal estimates are improved measurements of land area for each class based on error found in the map.

The first step in accuracy assessment is developing a stratified random sample. First, the total number of points $(N)$ is determined based on multinomial probability theory. The equation is shown below (Equation 1), where $\Pi \mathrm{i}$ is the proportion of a population in the ith class out of $k$ classes that has the proportion closest to $50 \%, B$ is the upper $(\alpha / \mathrm{k}) \times 100$ th percentile of the chi

$$
N=\frac{B \Pi_{i}\left(1-\Pi_{i}\right)}{b_{i}^{2}}
$$

Equation 1. Multinomial probability-based equation to calculate the number of stratified random sampling points necessary for a statistically-robust sample amount square $(\chi 2)$ distribution with 1 degree of freedom, $k$ is the number of land cover classes, and $b$ is the tolerable error or precision. A precision of 0.05 was selected.

After the accuracy assessment points are generated, the ground-truth condition was inferred based upon a combination of the UAS, Google Earth imagery, and expert interpretation of the original WorldView-2 and Pleiades imagery (Congalton and Green 2008). A confusion matrix is then generated from the accuracy assessment points, which shows the number of correctly classified pixels for each of the land cover change types. It also shows the incorrectly classified pixels and which class they were confused with. This information is useful to improve upon future iterations of a land cover map or to gain quantifiable insight into the map's quality, considering that all maps have some amount of error.

Using a confusion matrix, user's accuracy (1 - commission error), producer's accuracy (1 - omission error), and overall map accuracy are reported. Then, the accuracy metrics are adjusted based on unbiased areal proportions as described in Olofsson et al. (2014). Each accuracy-adjusted metric has an uncertainty estimate using a $95 \%$ confidence interval. The uncertainty metrics make class comparison more quantitative and precise (Olofsson et al. 2014). Based on the accuracy assessment results, unbiased areal estimates for each land cover class are derived. These estimates for each class essentially report a more refined estimate of the land area for each class because they account for the user's and producer's accuracies and associated confidence intervals.

Woerner. The progress and restoration of natural lands in the Woerner site have been most noticeable through the in-filling and re-vegetation of irrigation canals and drainage ditches. Because of this, the analysis focused on fine resolution of change in water-covered areas.

Progress in the Woerner site was determined \begin{tabular}{|c|c|c|}
\hline Change class & Area ha & $\begin{array}{c}\text { Percent of Study } \\
\text { Area }\end{array}$ \\
\hline Water to Vegetation & 31.1 & $2.5 \%$ \\
\hline Vegetation to Water & 13.3 & $1.1 \%$ \\
Persisting water & 9.6 & $0.7 \%$ \\
Other land areas & 1226 & $95.8 \%$ \\
Woerner Total area & 1280 & $100 \%$ \\
\hline
\end{tabular}

Table 1. Change between the 2010 and 2017 winter imagery for the Woerner site. 
support vector machine algorithm and training data derived from UAS imagery in conjunction with the WorldView-2 and Pleiades. It was observed that a large number of irrigation and drainage canals transitioned to vegetation in 2017 due to the restoration efforts (Figure 7). As shown in Table 1, approximately 31 hectares (ha) of water were converted into vegetation, whereas approximately 13 ha of vegetation were converted into water. Some of this is likely to be interannual variation not a part of this scoping study but which can be accounted for with a time-series of imagery.

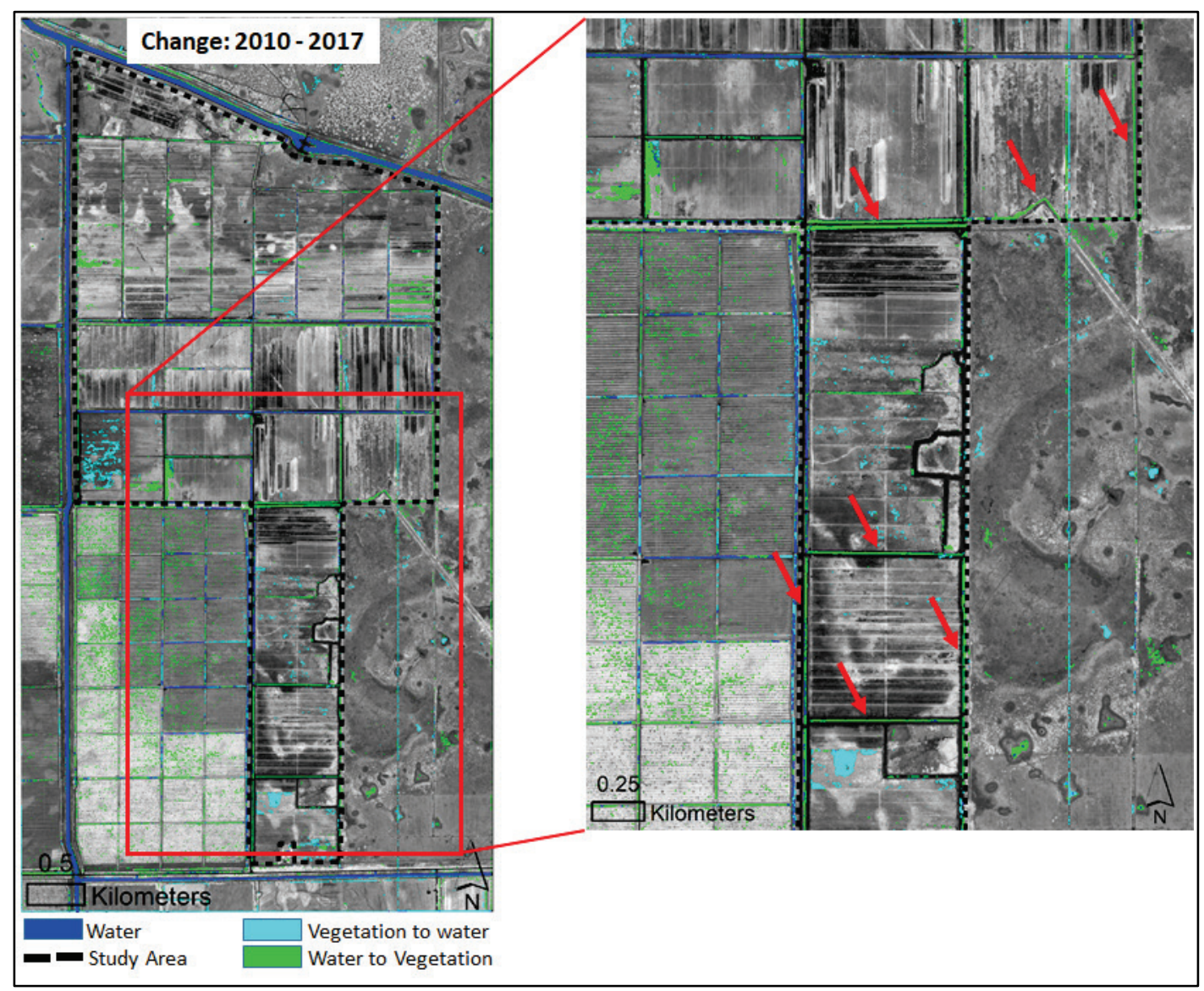

Figure 7. Areas of persisting water, and change between vegetation and water are shown based on images obtained during December 2010 and January 2017 and overlaid on a $2017 \mathrm{NDVI}$. Areas of change to note especially include along irrigation canals that have been converted into vegetation as seen in the zoomed image.

(C) 2015 DigitalGlobe NextView License

It is anticipated that UAS or high-resolution satellite imagery will observe more vegetative growth in the near future as canals are filled and promote hydrological changes.

The results of the multinomial probability equation suggested a sample size of approximately 60 points, which were stratified based upon the relative proportion of each land cover class (Congalton and Green 2008). Ideally, each class should have a minimum of 25 points for best results; however, in this case additional points were not added as it is not critical to the objectives of this work. The stratified random sampling points overlaid on an NDVI change image for 2010-2017 are shown in Figure A-2 the appendix. 
The confusion matrix is shown in Table A-1. In this study, if accuracy assessment procedures conducted in other studies (which does not use adjusted metrics) were followed, producer's accuracy for unchanged areas $(98.8 \% \pm 0.33 \%$ vs. $87.6 \%)$ and water-vegetation $(92.2 \% \pm 5.6 \%$ vs. $80.0 \%)$ would have been lower reported.

The results of area adjustment are shown in Figure A-3 for the original mapped areas (blue bars) and adjusted areas (orange bars) with 95\% confidence interval ranges shown overlaying the adjusted area. It is possible to reduce the range with more sampling points. It was found that overall, there may have been a slight underestimation in vegetation which changed to water, as well as overestimation in water changing to vegetation, and overestimation in water. However, based on the confidence interval ranges, it is not fully certain until more sampling points are obtained.

Blue Head. The Blue Head site analysis resulted in a vegetation change detection product and a land cover map for 2015. The 2015 land cover map is shown in Figure 8 and change detection image in Figure 9. The majority of change detected between 2011 and 2015 at Blue Head consisted of scrubtype cover transitioning to herbaceous wetland in the northeast portion of the study site, improved pasture transitioning to scrub-type cover in the southwest, and small gains in tree cover throughout the study site (Figure 9). Insets of the NDVI change detection are shown in Figure A-4 and Figure A-5 of the appendix. The accuracy assessment was only conducted on the land cover map. Using the multinomial probability approach described above, a total sample size of 340 points were generated in ENVI using a stratified random sample scheme with extra samples added to classes that were underrepresented, namely barren and open water. Using a support vector machine classification algorithm, the final land cover map represented eight distinct class types:

1. Barren - bare surface, unimproved road, built-up

2. Seasonal Wetlands - perennial submerged/semi-submerged macrophytes

3. Open Water - permanent water bodies

4. Seasonal Water - perennial pools and water holding depressions

5. Emergent Herbaceous Wetland - rooted, herbaceous hydrophytes

6. Pasture - land improved for pasture/sod farming

7. Grassland/Herbaceous - open grassland/scrub/low lying vegetation

8. Mixed Forest - composed of sabal palm, oak hammock, and pine stands. 


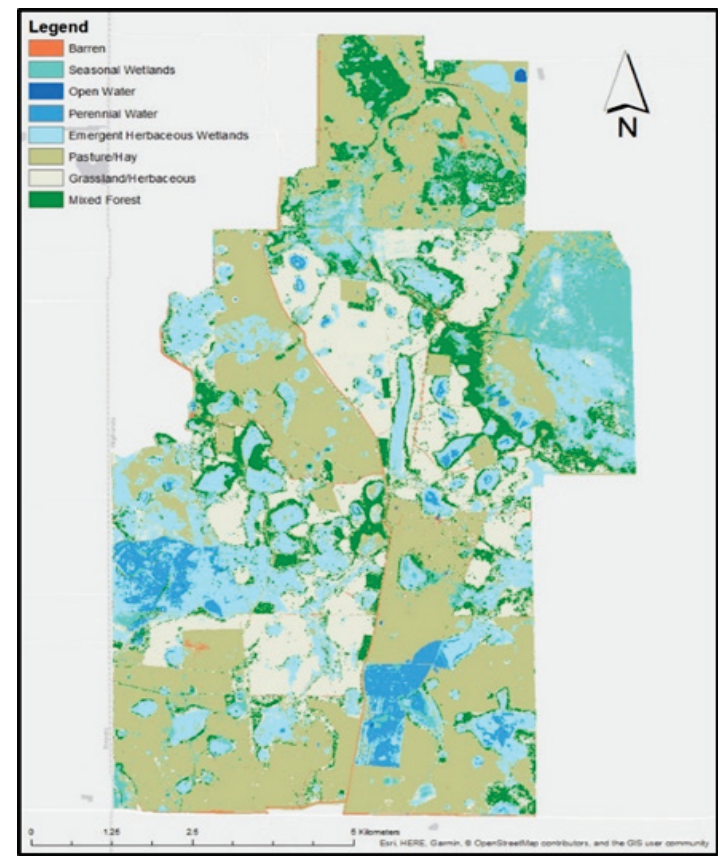

Figure 8. Classified map representing the land cover types of the Blue Head site, 2015.

(c) 2015 DigitalGlobe NextView License

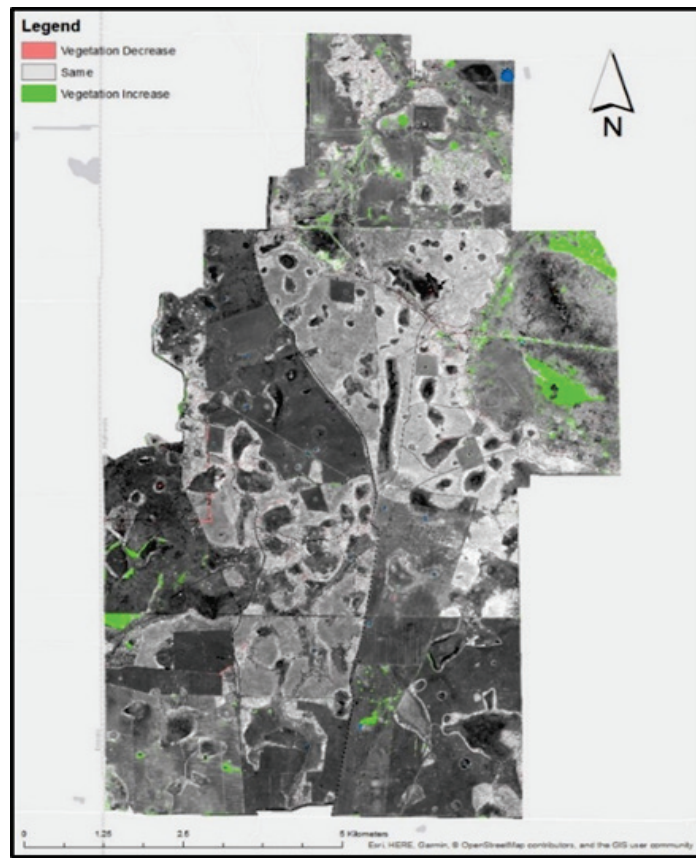

Figure 9. Change detection map representing change in vegetation cover between 2011 and 2015.

(C) 2015 DigitalGlobe NextView License

The areal adjusted overall accuracy was 0.81 . Full error matrix can be found in Table A-2 in the appendix. The vegetation change analysis was conducted by subtracting 2011 NDVI from 2015 NDVI of similar season. The difference product was then classified in classes of significant change by thresholding the histogram beyond two standard deviations from the mean. The results showed an increase of approximately 120 ha and a decrease of 28 ha as shown in Figures A-4 and A-5 in the appendix.

Hog Island. The Hog Island site analysis resulted in a multitemporal land cover change map between 2010 and 2015. To reduce compounded error from assessing the difference between separate 2010 and 2015 land cover maps, training data were developed specifically for the transition areas using a multitemporal image composite. The final land cover map is represented by five distinct class types and three change classes (Figure 10):

1. Barren - bare surface, unimproved road, built-up

2. Improved Pasture - land improved for pasture/sod farming

3. Grassland/Herbaceous - open grassland/scrub/low lying vegetation

4. Mixed Forest - composed of sabal palm, oak hammock, and pine stands

5. Citrus - citrus orchard

6. Wetland Gain - increase in wetland cover from 2010 to 2015

7. Wetland Loss - decrease in wetland cover from 2010 to 2015

8. Wetland Persistence - no change in wetland cover. 
The approximate area of change includes the following:

1. Wetland gain - 176 ha or $33 \%$ of the study site

2. Wetland loss - 12 ha or $2.3 \%$ of the study site

3. Wetland persistence -7 ha or $1.3 \%$ of the study site.

The map accuracy assessment resulted in an area-adjusted overall accuracy of 0.87 . Full error matrix can be found in Table A-3 in the appendix.

DISCUSSION: Throughout each of the ecological restoration demonstration sites, we successfully mapped landscape changes attributed to restoration efforts. The study benefited from combining UAS with

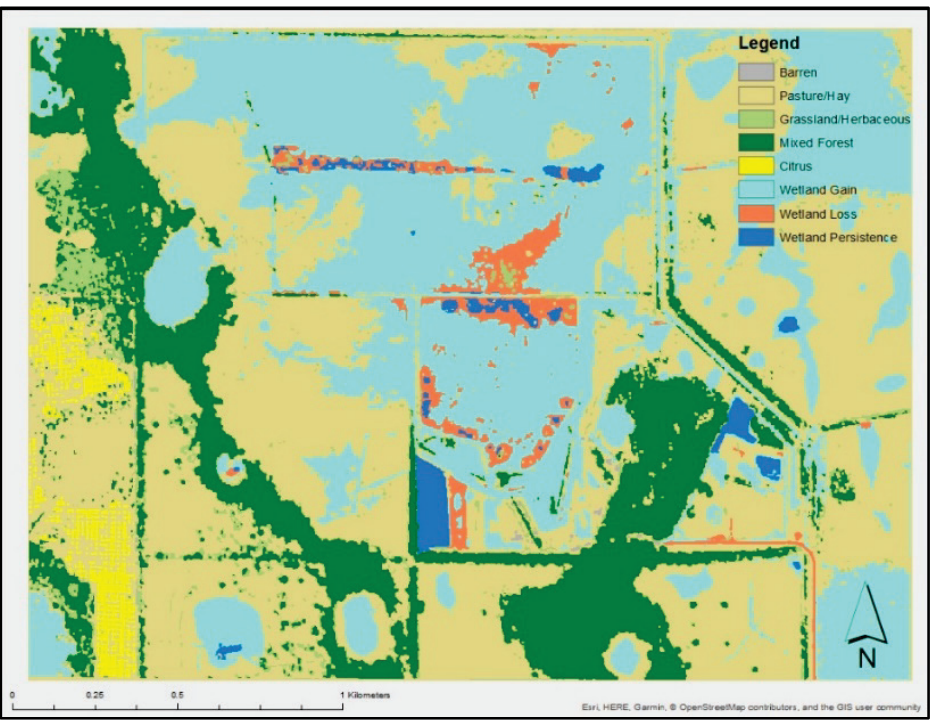

Figure 10. Hog Island classified map representing land cover change.

(c) 2015 DigitalGlobe NextView License satellite imagery and a machinelearning classification algorithm for ecological restoration mapping. However, several aspects remain that could significantly improve the state of research.

Improvements could be made by incorporating UAS technologies that collect multispectral imagery using well-calibrated geolocation sensors and spectral bands that extend beyond just visible colors. NIR and shortwave-infrared are critical for differentiating between land cover types, especially discriminating amongst specific vegetation types. They also offer improved mapping of water covered surfaces. On another note, satellite imagery acquired over this region often suffers from cloud contamination, which hinders land cover mapping. Fortunately, UAS is able to overcome thisbut impractical for systematically acquiring imagery (e.g., every 2 weeks). Therefore, one system that should be incorporated into the analysis is Synthetic Aperture Radar (SAR). SAR systems operate using microwaves, which can generally pass through atmospheric conditions such as cloud cover and consistently observe ground conditions on a regular basis. Implementation of satellite-based SAR such as Sentinel-1, which observes at C-band with a two satellite constellation achieving 6-12 day repeat overpass times, would be very beneficial to incorporate for vegetation change monitoring in the future.

SUMMARY: This study demonstrated a practical methodology that explicitly applied UAS and high spatial resolution satellite imagery for mapping land cover changes in land areas undergoing ecological restoration to natural habitat. A robust machine-learning algorithm, support vector machine, was used to classify time series of satellite and UAS imagery to monitor restoration of key demonstration sites. Though centimeter-scale UAS has areal coverage limitations, the combination of wide, synoptic coverage of satellite-acquired imagery with extremely fine resolution UAS useful for ground reference and supervised image classification training was leveraged. The synthesis of multiple sources of imagery and data prove critical to land cover mapping applications, especially considering environmental constraints and monitoring criteria such as cloud cover, seasonal land cover variation, fine-resolution landscape changes, and more, which are found in ecological restoration applications. 
In addition to land cover mapping for restoration of Florida wetlands to natural habitat, this study demonstrated the importance of a statistically robust accuracy assessment using multinomial probability sampling. Considering that no map is perfect, that the resulting areal adjusted and unbiased areal metrics can better inform decision makers about any given map's strengths or weaknesses. In the future, ecological restoration monitoring efforts should not only include methods and imagery from this study but also more spectrally robust UAS, SAR, and dense time-series stacks of moderate-fine resolution satellite imagery for outstanding results and improved species identification.

ADDITIONAL INFORMATION: This technical note was prepared by Sean Griffin and Kristofer Lasko, GRL ERDC. The UAS imagery was collected by Victor Wilhelm, USACE, Jacksonville District. Helpful feedback was provided by Molly Reif and Glenn Suir. This technical note should be cited as follows:

Griffin, S. P., and K. Lasko. 2020. Using Unmanned Aircraft System (UAS) and Satellite Imagery to Map Aquatic and Terrestrial Vegetation. ERDC/GRL TN-20-4. Vicksburg, MS: US Army Engineer Research and Development Center. http://dx.doi.org/10.21079/11681/38086

\section{REFERENCES}

Agisoft Photoscan Professional Edition, Version 1.2. https://www.agisoft.com/

Berstein, L. S., S. M. Adler-Golden, X. Jin, B. Gregor, and R. L. Sundberg. 2012. "Quick Atmospheric Correction (QUAC) Code for VNIR-SWIR Spectral Imagery: Algorithm Details." $20124^{\text {th }}$ Workshop on Hyperspectral Image and Signal Processing: Evolution in Remote Sensing (WHISPERS).

Congalton, R.G., and K. Green. 2008. Assessing the Accuracy of Remotely Sensed Data: Principles and Practices. Boca Raton, FL: CRC Press.

Griffin S., J. Rogan, D. M. Runfola. 2011. "Application of Spectral and Environmental Variables to Map the Kissimmee Prairie Ecosystem Using Classification Trees.” GIScience \& Remote Sensing 48: 299-323.

Kontgis, C., A. Schneider, and M. Ozdogan. 2015. "Mapping Rice Paddy Extent and Intensification in the Vietnamese Mekong River Delta with Dense Time Stacks of Landsat Data." Remote Sensing of Environment 169: 255-269.

Lasko, K., K. P. Vadrevu, V. T. Tran, and C. Justice. 2018. "Mapping Double and Single Crop Paddy Rice with Sentinel$1 \mathrm{~A}$ at Varying Spatial Scales and Polarizations in Hanoi, Vietnam." IEEE Journal of Selected Topics in Applied Earth Observations and Remote Sensing 11(2): 498-512.

Manjula, K. R., J. Singaraju, and A. K. Varma. 2013. "Data Preprocessing in Multi-Temporal Remote Sensing Data for Deforestation Analysis." Global Journal of Computer Science and Technology Software and Data Engineering 13(6): 19-25.

Mondal, A., S. Kundu, S. K. Chandniha, R. Shukla, and P. K. Mishra. 2012. "Comparison of Support Vector Machine and Maximum Likelihood Classification Technique Using Satellite Imagery." International Journal of Remote Sensing and GIS 1(2): 116-123.

Olofsson, P., G. M. Foody, M. Herold, S. V. Stehman, C. E. Woodcock, and M. A. Wulder. 2014. "Good Practices for Estimating Area and Assessing Accuracy of Land Change.” Remote Sensing of Environment 148: 42-57.

Reif, M. K., and H. J. Theel. 2017. "Remote Sensing for Restoration Ecology: Application for Restoring Degraded, Damaged, Transformed, or Destroyed Ecosystems." Integrated Environmental Assessment and Management 13(4): 614-630.

Tucker, C. J. 1979. "Red and Photographic Infrared Linear Combinations for Monitoring Vegetation.” Remote Sensing of Environment 8(2): 127-150. 


\section{APPENDIX}

\section{SATELLITE IMAGERY DATA PRE-PROCESSING}

\section{Imagery Data}

ETP Tools is currently developed to ingest any DigitalGlobe dataset with IMD header and RPB metadata containing the rational polynomial coefficients (RPCs).

\section{Geometric Correction}

The main steps to geometric correction are orthorectification and image-to-image coregistration. An orthorectified image (or orthophoto) is one where each pixel represents a true ground location and all geometric, terrain, and sensor distortions have been removed to within a specified accuracy. Orthorectification transforms the central perspective of an aerial photograph or satellite-derived image to an orthogonal view of the ground, which removes the effects of sensor tilt and terrain relief. Scale is constant throughout the orthophoto, regardless of elevation, thus providing accurate measurements of distance and direction. The most accurate orthorectification requires a set of RPCs, coordinates that match the image point to the ground point. Most commercial sensors are provided with RPC metadata, which are readily ingested and parsed by the ENVI and ArcGIS modules.

Image coregistration is performed when the intention is to study two or more images in a temporal series, typically to assess change. Although orthorectification can georeference images within high geometric accuracy, this does not guarantee that an image series aligns to an acceptable degree of error. ENVI and ArcGIS both offer cross-correlation correction by linking similar spectral features in the images or mutual information for fusing data of different modalities such as optical, radar, and LiDAR. These automated methods provide high coregistration accuracy unless one or both images are highly affected by cloud, cloud shadow, or complex terrain relief.

\section{Radiometric Correction}

Radiometric correction is performed to reduce or correct errors in the digital numbers of images and is a prerequisite for comparing a temporal series. Correction includes radiance calibration and atmospheric correction. Calibration applies a set of gain and offset coefficients provided in the sensor metadata to convert digital number counts to real world units of at-sensor radiance (i.e., above atmosphere). Atmospheric correction reduces the effects of scattering and absorption in the atmosphere using a variety of methods that include physical and empirical models, and image-based and cross-sensor techniques. Although physical-based first-principles methods can produce accurate estimates of surface reflectance (e.g., Fast Line-of-sight Atmospheric Analysis of Hypercubes, or FLAASH), they require specialized software that can be cost prohibitive and computationally intensive. With consideration to EMRRP, image-based techniques were chosen over physical models for several reasons: they are readily available in most commercial software packages, generally sensor agnostic, applied by practitioners of any skill level, and studies have shown that image-based techniques can estimate surface reflectance within a small margin of error from ground-truth spectra. This study applied the Quick Atmospheric Correction (QUAC) algorithm that derives correction parameters directly from the observed pixel spectra within the image scene at a fraction of the time compared to FLAASH (Berstein et al. 2012). Also, unlike FLAASH, QUAC requires no 
parameterization to apply. The Figure A-1 compares QUAC and FLAASH, showing minimal difference between the image and physical model based techniques.

Image acquisition dates for each study site includes the following:

1. Woerner
a. 2010-12-16 (Pleiades)
b. 2017-01-31 (Pleiades)

2. Blue Head
a. 2010-12-17 (Worldview-2)
b. 2011-01-14 (Worldview-2)
c. 2015-02-11 (Pleiades)
d. 2015-02-14 (Worldview-2)
e. 2015-10-30 (Pleiades)

3. Hog Island
a. 2010-12-17 (Worldview-2)
b. 2011-01-14 (Worldview-2)
c. 2014-10-24 (Pleiades)
d. 2015-02-14 (Worldview-2)
e. 2015-04-04 (Pleiades)
f. 2015-10-30 (Pleiades) 
ERDC/GRL TN-20-4

September 2020
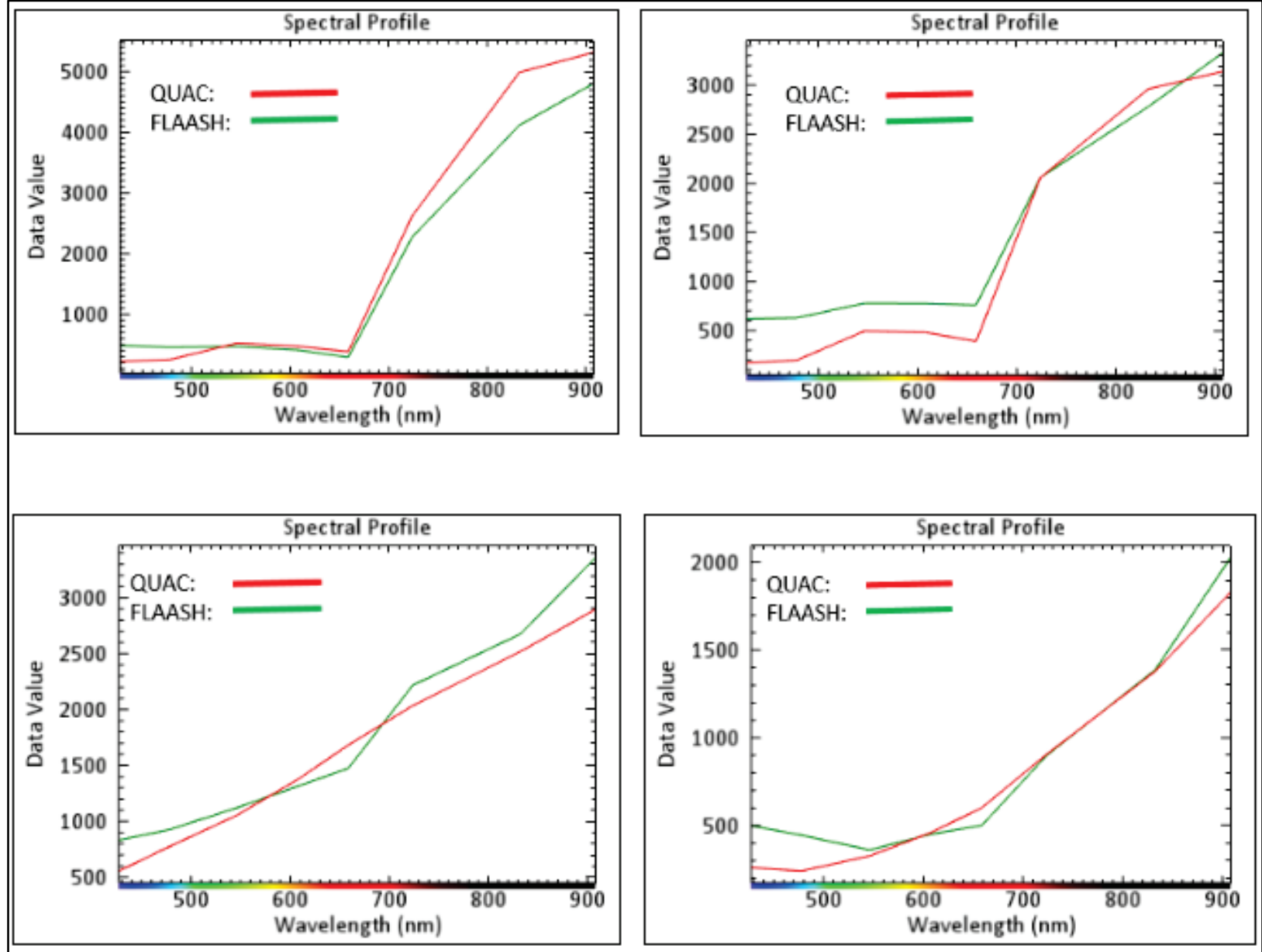

Figure A-1. Comparison of atmospheric correction techniques highlighting minimal difference between the types.

4. Spectral Indices

Spectral indices are data transforms that combine the spectral reflectance of two or more wavelengths to enhance features of interest such as vegetation, water, impervious surfaces, and burned areas. Spectral indices can also serve as ancillary data to improve map classification accuracy. ETP Tools can automatically calculate a variety of indices based on the wavelength and band width stored in the sensor metadata and image header. 


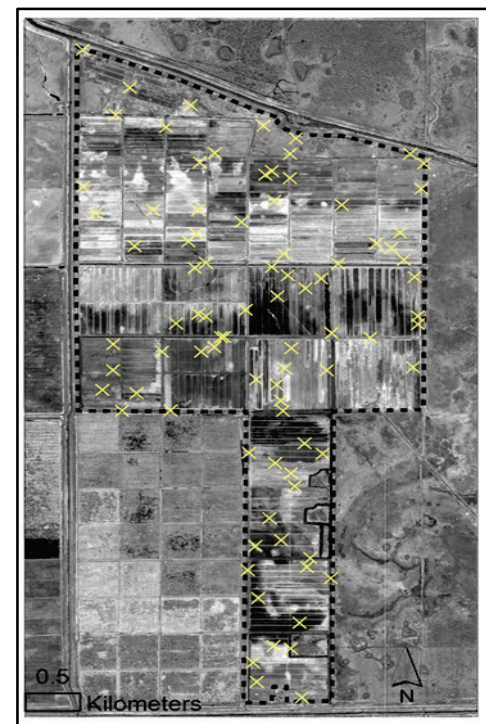

Stratified Random Sampling Point

Figure A-2. Stratified random sampling points for Woerner study area.

Table A-1. Confusion matrix for the land cover change map and the reference data (UAS, WV-2, Google Earth). The classes are water-water, water-vegetation, vegetation-water, and unchanged. The number of pixels sampled for each class is shown in the top table. The bottom table shows the unbiased areal proportions and adjusted accuracy metrics with $95 \%$ confidence intervals.

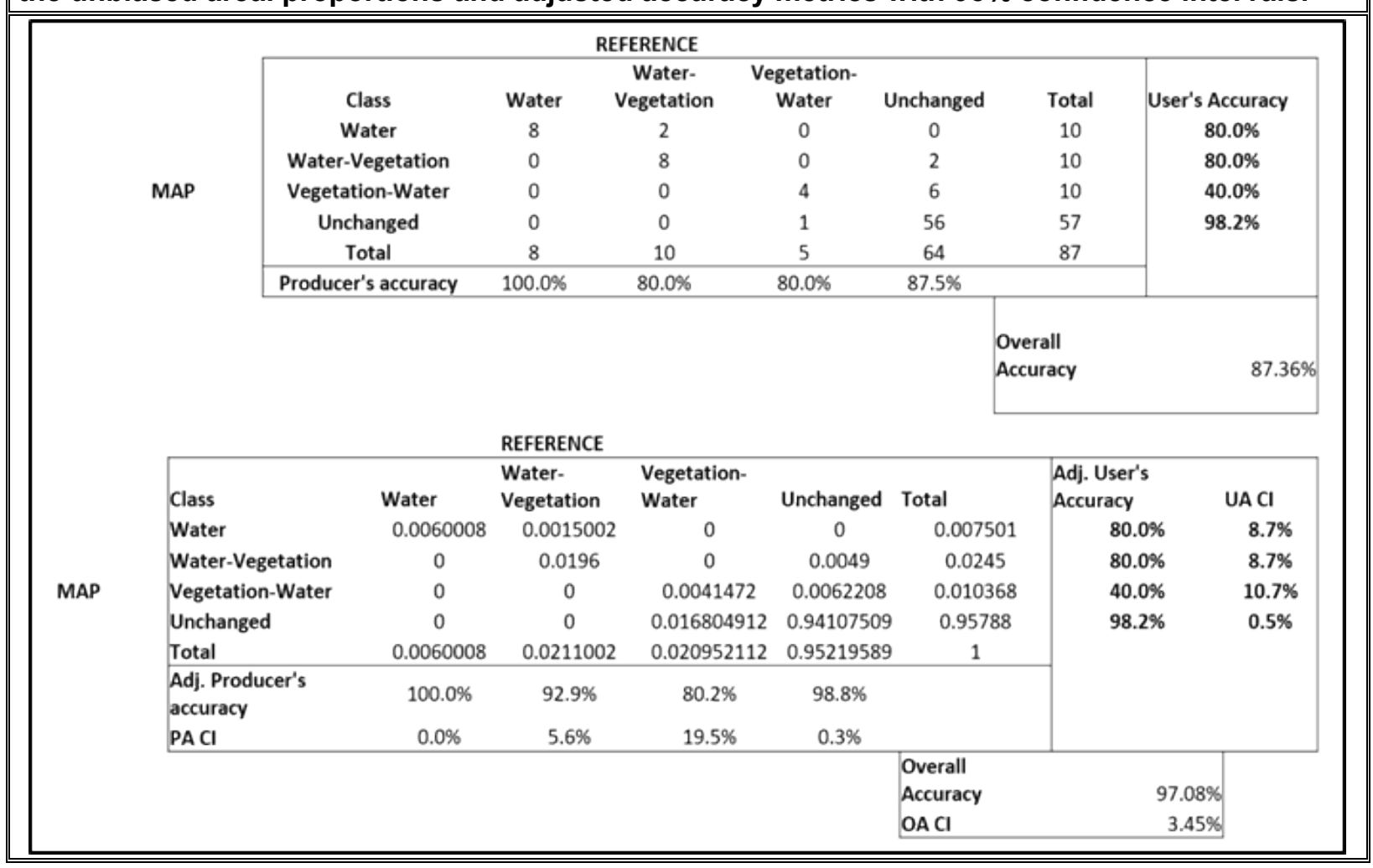




\section{Land Cover Accuracy- Adjustment}

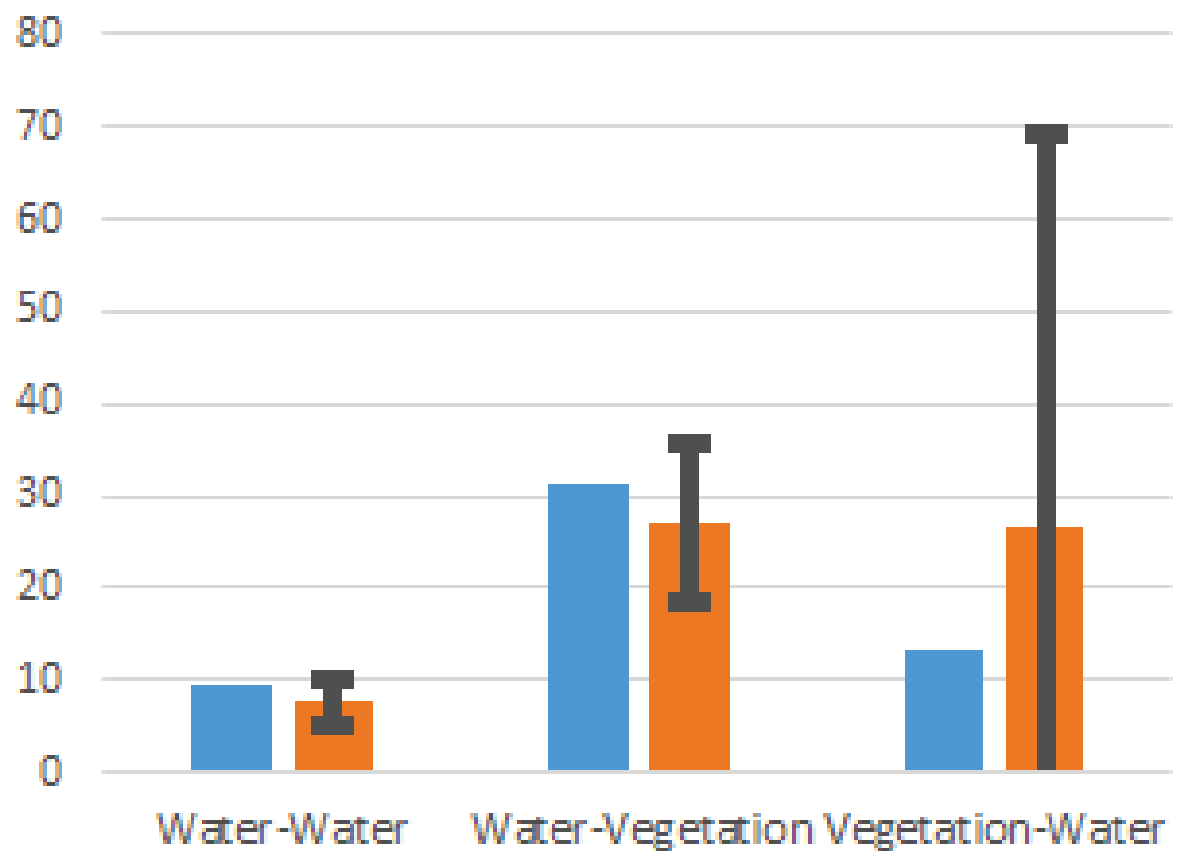

\section{Mapped area a Accur acy-adjusted area}

Figure A-3. Areas for each land cover class are adjusted based on the results of the accuracy assessment. The accuracy-adjusted areas provide the best estimate of land area for each class. The $95 \%$ confidence interval ranges are shown in the error bars. Error ranges can be reduced with more sampling points. 


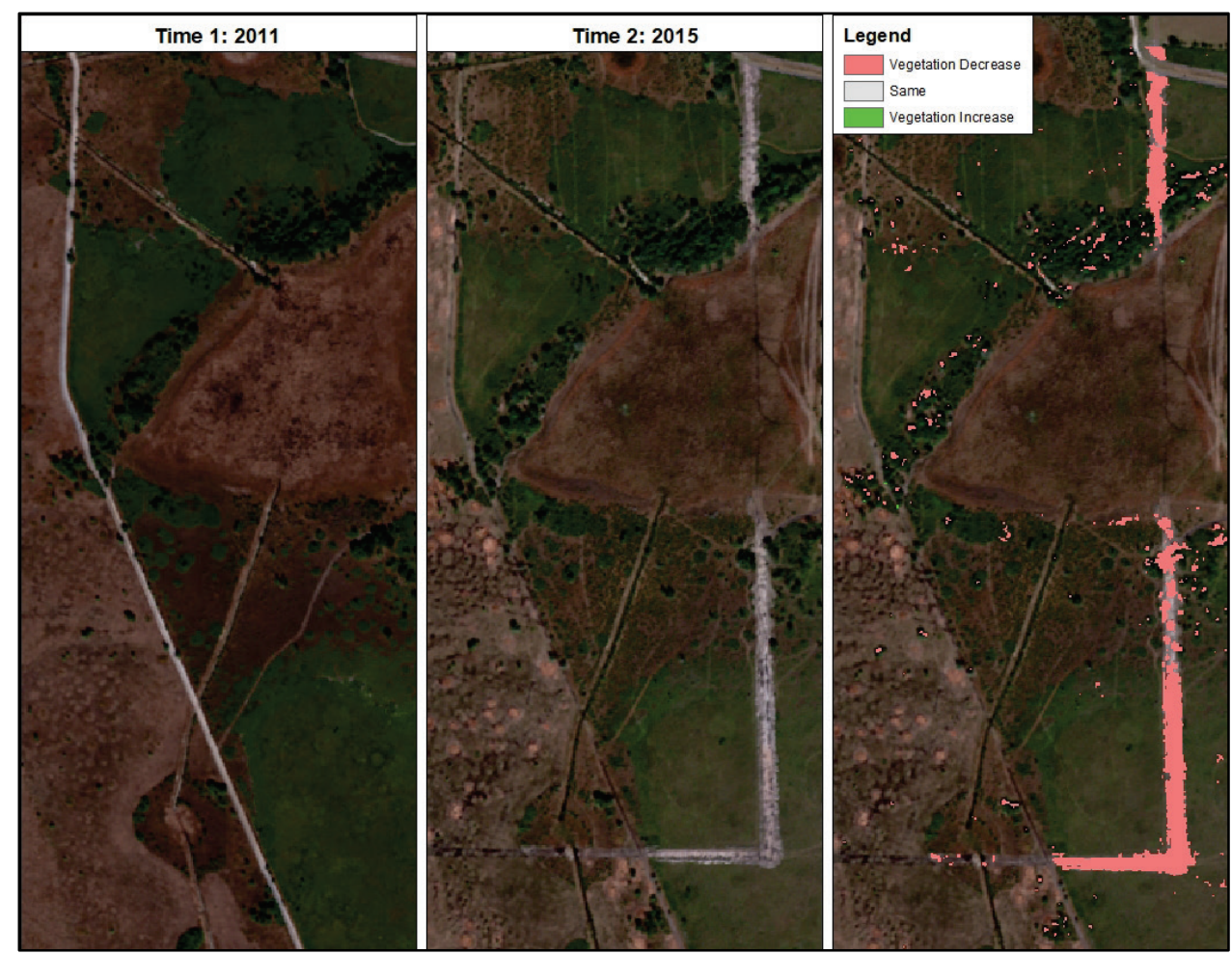

Figure A-4. Example of vegetation decrease derived from NDVI change analysis over the Blue Head site (anthropogenic change).

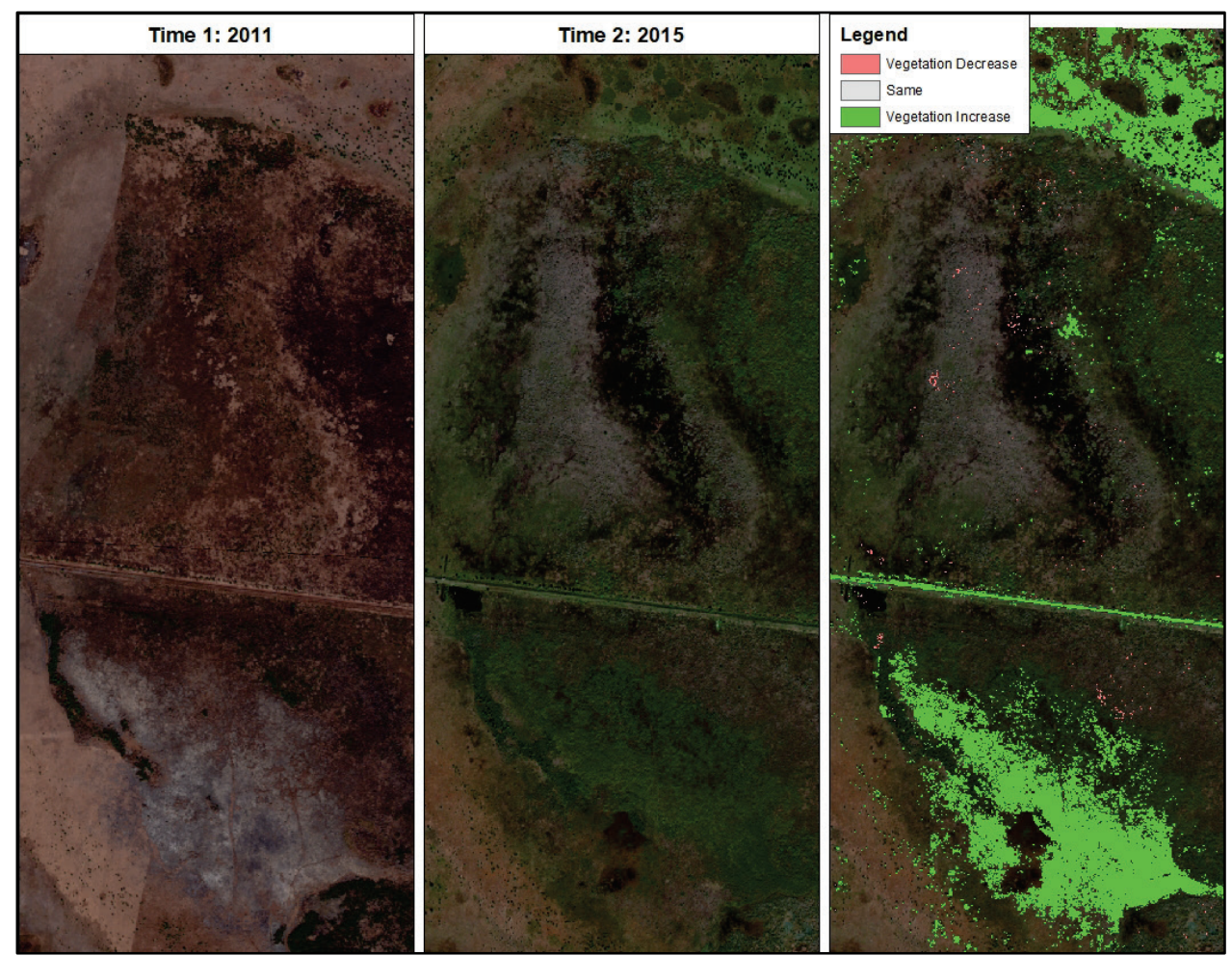

Figure A-5. Example of vegetation Increase derived from NDVI change analysis over the Blue Head site (Transition to wetland). 
Table A-2. Standard confusion matrix with the top table showing pixel counts for each land cover class and the bottom table showing unbiased area proportions and accuracy metrics derived for Blue Head study site.

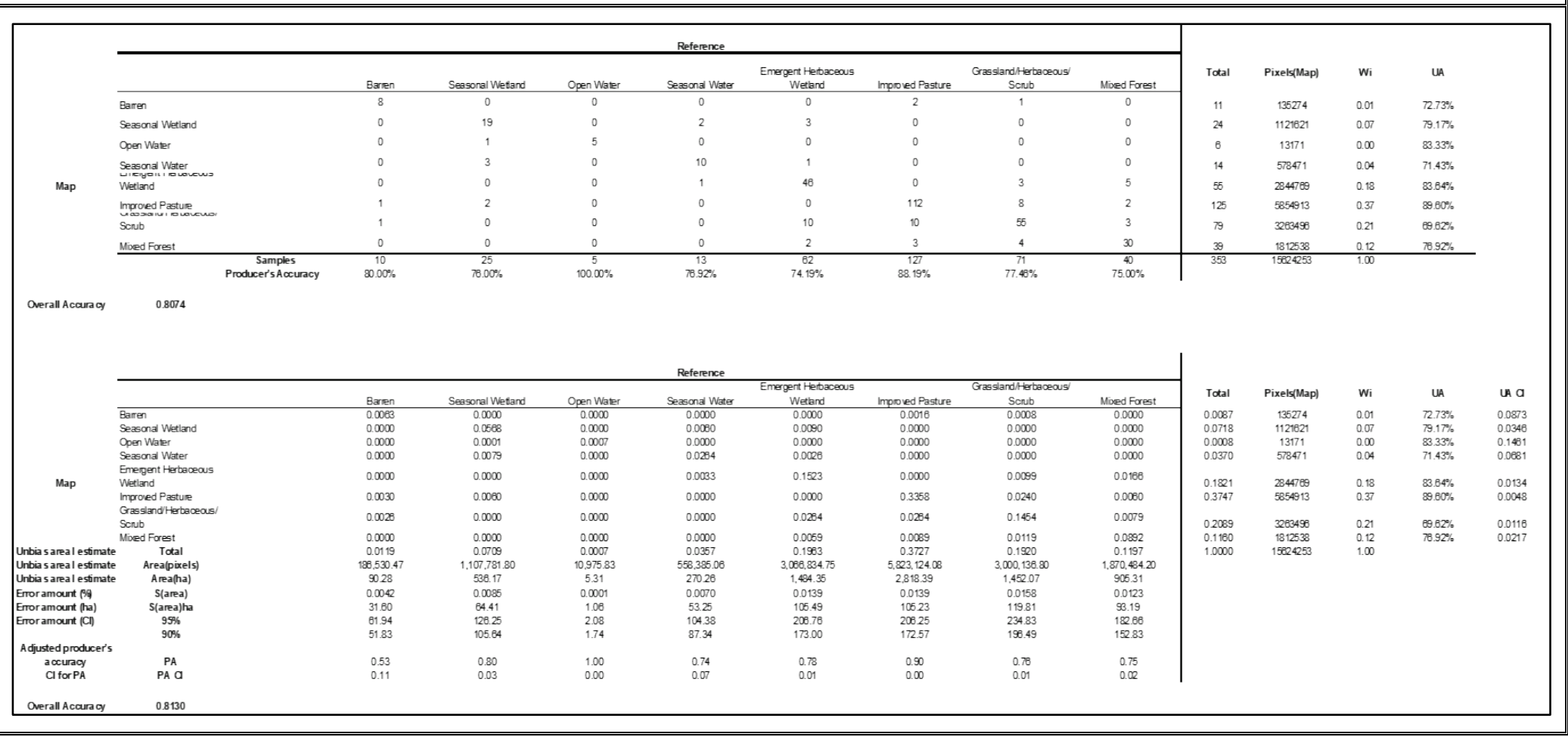




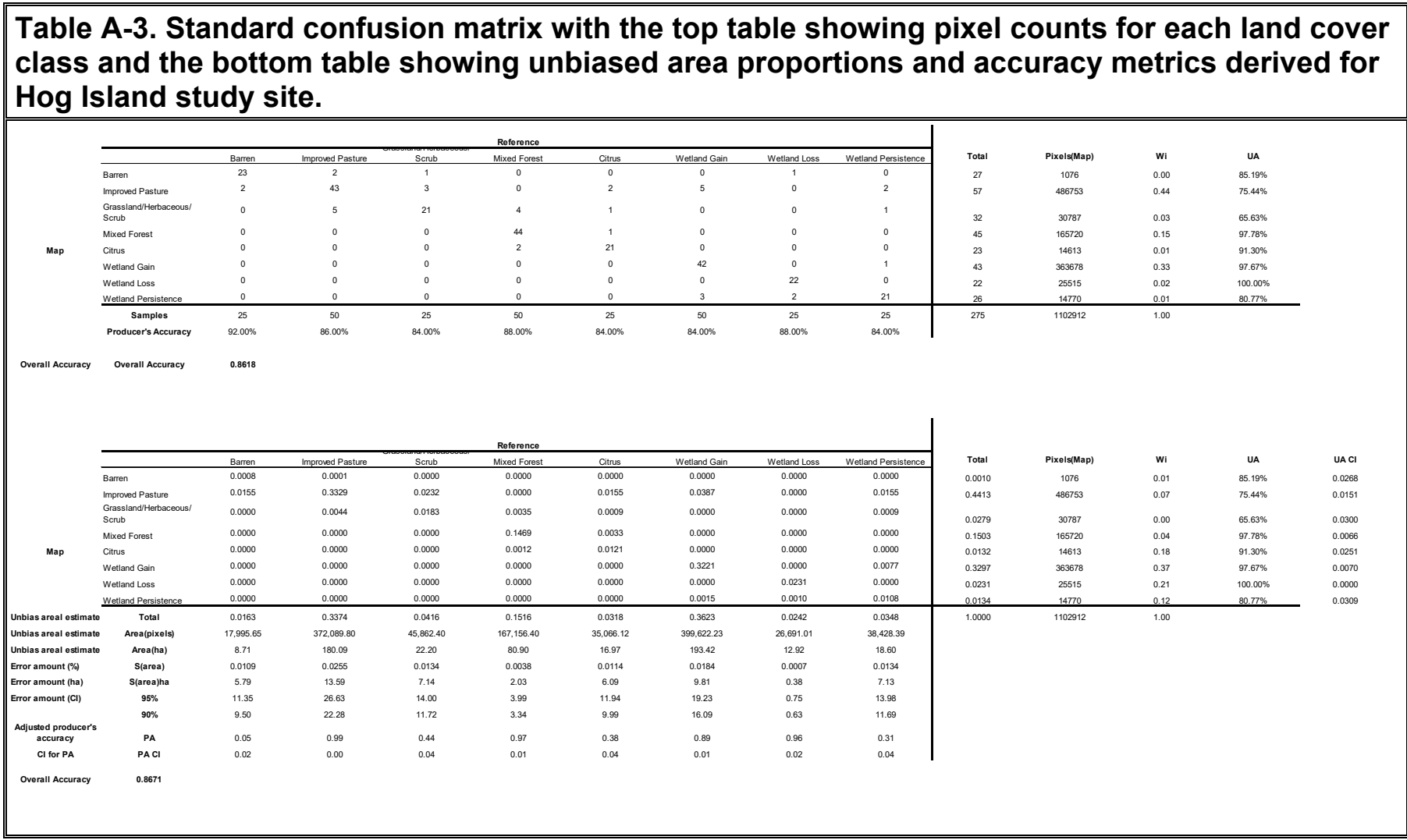


ERDC/GRL TN-20-4

September 2020

NOTE: The contents of this technical note are not to be used for advertising, publication, or promotional purposes. Citation of trade names does not constitute an official endorsement or approval of the use of such products. 